\title{
Determination of Magnetic Exchange Stiffness and Surface Anisotropy Constants in Epitaxial Ni_ $\{1-x\}$ Co_ $\{x\}(001)$ Films
}

P. Talagala

Wayne State University

Petru S. Fodor

Cleveland State University, p.fodor@csuohio.edu

Haddad R. Naik

Wayne State University

Lowell E. Wenger

Wayne State University, wenger@physics.wayne.edu

P. P. Vaishnava

Kettering University

Follow this and additional works at: https://engagedscholarship.csuohio.edu/sciphysics_facpub

ipraxtopala fortyadditionalyauthors

How does access to this work benefit you? Let us know!

Publisher's Statement

(C2002 American Physical Society

\section{Repository Citation}

Talagala, P.; Fodor, Petru S.; Naik, Haddad R.; Wenger, Lowell E.; Vaishnava, P. P.; and Naik, V. M., "Determination of Magnetic Exchange Stiffness and Surface Anisotropy Constants in Epitaxial Ni_ $\{1-\mathrm{x}\}$ Co_ $\{x\}(001)$ Films" (2002). Physics Faculty Publications. 200.

https://engagedscholarship.csuohio.edu/sciphysics_facpub/200

This Article is brought to you for free and open access by the Physics Department at EngagedScholarship@CSU. It has been accepted for inclusion in Physics Faculty Publications by an authorized administrator of EngagedScholarship@CSU. For more information, please contact library.es@csuohio.edu. 
Authors

P. Talagala, Petru S. Fodor, Haddad R. Naik, Lowell E. Wenger, P. P. Vaishnava, and V. M. Naik 


\title{
Determination of magnetic exchange stiffness and surface anisotropy constants in epitaxial $\mathrm{Ni}_{1-x} \mathrm{Co}_{x}(001)$ films
}

\author{
P. Talagala, P. S. Fodor, D. Haddad, R. Naik, and L. E. Wenger \\ Department of Physics and Astronomy, Wayne State University, Detroit, Michigan 48201 \\ P. P. Vaishnava \\ Department of Science and Mathematics, Kettering University, Flint, Michigan 48504 \\ V. M. Naik \\ Department of Natural Sciences, University of Michigan-Dearborn, Dearborn, Michigan 48128 \\ (Received 22 January 2002; published 31 October 2002)
}

\begin{abstract}
Magnetic characteristics of epitaxial $\mathrm{Ni}_{1-x} \mathrm{Co}_{x}(001)(x=0,0.16$, and 0.50$)$ films with nominal $200 \mathrm{~nm}$ thickness on $\mathrm{Cu}(001) / \mathrm{Si}(100)$ substrates have been investigated by magnetization and ferromagnetic resonance measurements in order to better clarify the rationale for the large variation in the magnetic exchange stiffness constant $A$, previously determined from different measurements. The exchange constant as well as the saturation magnetization, effective demagnetizing field, fourth-order magnetocrystalline, and second-order perpendicular uniaxial magnetic anisotropy fields has been determined. The analyses of low-temperature saturation magnetization data on these films yield $A$ values that increase from $0.82 \times 10^{-6} \mathrm{erg} / \mathrm{cm}$ for a pure $\mathrm{Ni}$ film to $2.27 \times 10^{-6} \mathrm{erg} / \mathrm{cm}$ for the $\mathrm{Ni}_{0.50} \mathrm{Co}_{0.50}$ film. Furthermore, spin-wave resonance volume modes observed in $x$ $=0$ and 0.16 films indicate that the surface plays a role in the exchange stiffness constant determination as the surface anisotropy constants are found to be approximately 1 and $4 \mathrm{erg} / \mathrm{cm}^{2}$, respectively. The latter value is substantially larger than that for any other system reported so far.
\end{abstract}

DOI: 10.1103/PhysRevB.66.144426

PACS number(s): 75.70.Ak, 76.50.+g

\section{INTRODUCTION}

Detailed micromagnetic calculations of domain structures ${ }^{1}$ are of current interest in several areas of magnetism, e.g., the process of magnetization reversal in materials, the modeling of macroscopic magnetic properties, and the determination of magnetic properties of nanocrystalline ferromagnetics. ${ }^{2,3}$ One important materials parameter needed in these micromagnetic calculations is the exchange stiffness constant $A$. This exchange stiffness constant is related to the spin-wave stiffness constant $D$ through the relation $A$ $=D M_{s}(0) / 2 g \mu_{B}$, where $M_{s}(0)$ is the saturation magnetization at zero temperature, $g$ the spectroscopic $g$ factor, and $\mu_{B}$ the Bohr magneton. ${ }^{3}$ Both experimental ${ }^{4-8}$ and theoretical ${ }^{9,10}$ determinations of $D$ have been reported for Ni, Co, and their alloys, four of which are presented in Fig. 1. Clearly, the results from these studies show a large variation in $D$ as well as in their Co concentration dependence. The early magnetization study by Maeda et al. ${ }^{5}$ on fcc $\mathrm{Ni}_{1-x} \mathrm{Co}_{x}$ bulk alloys (0.1-mm-thick platelets) using a pendulum-type magnetometer finds $D$ values close to $400 \mathrm{meV} \AA^{2}$ with a minimum occurring at a Co content of 10 at. \%. A nearly identical Co concentration dependence is seen in the $D$ values determined from a spin-wave resonance (SWR) study of $\mathrm{Ni}_{1-x} \mathrm{Co}_{x}$ thin films (200-500 nm in thickness) by Cullis and Heath; ${ }^{4}$ however, the $D$ values for identical Co concentrations are $\sim 250$ $\mathrm{meV} \AA^{2}$ lower. In contrast, neutron inelastic scattering results reported by Mikke et al. ${ }^{6}$ find the $D$ values to be larger and with a different Co concentration dependence as the $D$ value for pure Ni is about $440 \mathrm{meV} \AA^{2}$, while the $D$ values for the alloys are between 550 and $600 \mathrm{meV} \AA^{2}$. It was noted by
Cullis and Heath ${ }^{4}$ that the difference might be the result of the magnetization and neutron scattering experiments dealing with traveling spin waves, while the SWR study dealt with standing spin waves in thin films where surfaces play an important role in the determination of $D$.

The aforementioned experimental determinations of $D$ can be contrasted to the band structure calculations of $D$ for fcc Ni-Co alloys by Wakoh ${ }^{10}$ as indicated by the dotted line in Fig. 1. As seen in Fig. 1, the theoretically calculated values of $D$ show an exponential-like, continuous decrease with increasing Co concentration over the entire Co range. This behavior is not consistent with any of the experimental results, even though the magnitude of $D$ is most similar to the $D$ values obtained from the SWR study. A more realistic comparison of $D$ values for the Ni-Co system would require

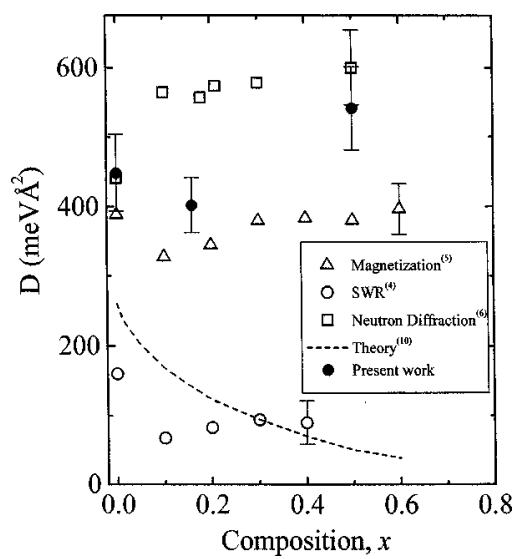

FIG. 1. Spin-wave stiffness constant $D$ as a function of Co composition for Ni-Co alloys. 
the inclusion of interatomic exchange interactions in the band structure calculations.

In order to provide further clarification of the magnetic exchange stiffness constant $A$ and the spin-wave stiffness constant $D$ for Ni-Co alloy films as well as on other magnetic parameters, the results from detailed magnetization and ferromagnetic resonance (FMR) measurements on three wellcharacterized epitaxial $\mathrm{Ni}_{1-x} \mathrm{Co}_{x}(001)$ thin films $(x$ $=0,0.16,0.50)$ are reported. The values of $A$ from analyses of the low-temperature-dependent saturation magnetization data are consistent with earlier magnetization ${ }^{5}$ and neutron diffraction results ${ }^{6}$ on bulk alloys. Furthermore, the observation of SWR volume modes in these thin-film samples indicates the presence of a surface anisotropy that should be taken into account when determining the magnetic exchange stiffness constants from SWR measurements.

\section{EXPERIMENT}

Film structures of $\mathrm{Cu} / \mathrm{Ni}_{1-x} \mathrm{Co}_{x} / \mathrm{Cu}(0 \leqslant x \leqslant 0.50)$ were prepared in an ultrahigh vacuum using a molecular beam epitaxy (MBE) deposition system operating at a base pressure of $2 \times 10^{-10}$ Torr. Epitaxial $\mathrm{Ni}_{1-x} \mathrm{Co}_{x}(001)$ films were grown on a 100 -nm-thick $\mathrm{Cu}(001)$ seed layer previously deposited on $\mathrm{Si}(001)$ substrates etched with a $10 \%$ hydrofluoric acid solution. Further details of the growth of the $\mathrm{Ni}(001)$ layers on $\mathrm{Cu}(001)$ layers as well as of the growth of $\mathrm{Cu}(001)$ seed layers on hydrogen-terminated $\mathrm{Si}(001)$ can be found in Ref. 11. All films were capped with a 5-nm-thick $\mathrm{Cu}$ layer for protection against oxidation and to provide symmetric interfaces for the alloy film. The deposition rate was maintained at $\sim 0.5 \AA / s$ with the films having a nominal thickness $d$ of $\sim 200 \mathrm{~nm}$. The thickness of the films was determined to within $\pm 5 \%$ from the quartz thickness monitor, which was previously calibrated using a diamond stylus profilometer. Regulating the deposition rates of cobalt and nickel during the codeposition of $\mathrm{Ni}$ and $\mathrm{Co}$ sets the composition for the $\mathrm{Ni}_{1-x} \mathrm{Co}_{x}$ films.

The epitaxial nature of the films was confirmed by monitoring the reflection high-energy electron diffraction (RHEED) patterns during the film growth as well as by standard x-ray diffraction (XRD) $\theta-2 \theta$ scans using $\mathrm{Cu} K \alpha$ radiation. As seen in Fig. 2, the XRD patterns exhibit only the (002) peaks indicative of epitaxial (001) growth. Using the bulk lattice constants for $\mathrm{Ni}$ and fcc $\mathrm{Co}$, the interatomic spacing $d_{002}$ should follow a linear behavior with increasing Co concentration according to Vegard's rule. Although a linear behavior is observed (see inset of Fig. 2), the shift in the experimental data from the expected linear behavior indicates the presence of a residual strain in the films.

A Quantum Design superconducting quantum interference device (SQUID) magnetometer (model MPMS-5S) was used to perform the magnetization measurements. The magnetic hysteresis loops were obtained at room temperature on all films with magnetic field orientations both normal and parallel to the film plane in order to determine the magnetic anisotropies of the films. Temperature-dependent saturation magnetization data were also acquired to determine the spinwave exchange stiffness constants as described in the next

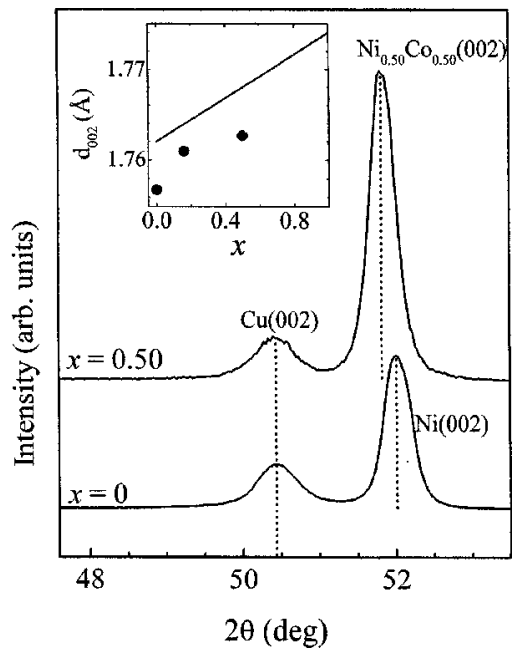

FIG. 2. X-ray diffraction scans for $\mathrm{Ni}_{0.50} \mathrm{Co}_{0.50}$ and $\mathrm{Ni}$ films. The inset displays the $d_{002}$ spacing as a function of the Co concentration $x$ with the solid line representing the concentration dependence based on Vegard's rule and the bulk lattice constants.

section. A 9.5-GHz $X$-band spectrometer was used to perform FMR/SWR measurements at room temperature using a standard lock-in detection technique. The sample (typical size of $4 \mathrm{~mm} \times 4 \mathrm{~mm}$ ) was mounted on a rod along the vertical axis of a $\mathrm{TE}_{101}$ rectangular resonance cavity with the capability to rotate the sample about a vertical axis to obtain the angular dependence. A horizontal dc magnetic field is provided by a 12 -in. Varian electromagnet that has a range of 0-19 kOe.

\section{ANALYSES AND DISCUSSION}

\section{A. Magnetization measurements}

Figure 3 shows the hysteresis loops for the $\mathrm{Ni}_{0.50} \mathrm{Co}_{0.50}(001)$ film, which is similar to the loops obtained for other two films. All loops clearly show that the easy axis of magnetization lies in the plane of the films. From these hystereses, the values of $M_{s}$ and $4 \pi M_{\text {eff }}$ (determined using the area between the parallel and perpendicular hysteresis

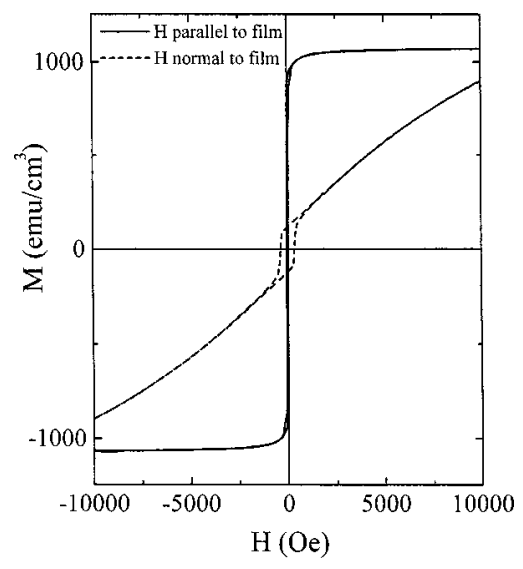

FIG. 3. $M$-vs- $H$ hysteresis loops for magnetic fields parallel and perpendicular to the plane of the $\mathrm{Ni}_{0.50} \mathrm{Co}_{0.50}$ film. 
TABLE I. Various magnetic parameters and constants determined from magnetization (Mag.), ferromagnetic resonance (FMR), and spin-wave resonance (SWR) measurements on $\mathrm{Ni}_{1-x} \mathrm{Co}_{x}$ thin-film samples of thickness $d . g=2.210,2.205$, and 2.195 for $x=0,0.16$, and 0.50 , respectively.

\begin{tabular}{|c|c|c|c|c|c|c|c|c|c|c|}
\hline \multicolumn{2}{|c|}{ Film sample } & \multirow{2}{*}{$\begin{array}{c}\begin{array}{c}M_{s} \\
\left(\mathrm{emu} / \mathrm{cm}^{3}\right)\end{array} \\
\text { Mag. }\end{array}$} & \multicolumn{2}{|c|}{$\begin{array}{c}4 \pi M_{\text {eff }} \\
(\mathrm{kG})\end{array}$} & \multirow{3}{*}{$\begin{array}{c}\begin{array}{c}H_{4} \\
(\mathrm{kOe})\end{array} \\
\text { FMR } \\
-0.2 \pm 0.1\end{array}$} & \multicolumn{2}{|c|}{$\begin{array}{c}H_{2} \\
(\mathrm{kOe})\end{array}$} & \multirow{3}{*}{$\begin{array}{c}A \\
\left(10^{-6} \mathrm{erg} / \mathrm{cm}\right) \\
\text { Mag. } \\
0.82 \pm 0.06\end{array}$} & \multirow{3}{*}{$\begin{array}{c}D \\
\left(\mathrm{meV}^{\circ} \AA^{2}\right) \\
\text { Mag. } \\
449 \pm 55\end{array}$} & \multirow{3}{*}{$\begin{array}{c}\begin{array}{c}K_{s} \\
\left(\mathrm{erg} / \mathrm{cm}^{2}\right)\end{array} \\
\mathrm{SWR} \\
0.93\end{array}$} \\
\hline $\mathrm{Ni}_{1-x} \mathrm{Co}_{x}$ & $d(\mathrm{~nm})$ & & Mag. & FMR & & Mag. & FMR & & & \\
\hline$x=0$ & $220 \pm 10$ & $470 \pm 35$ & $5.5 \pm 0.2$ & $5.3 \pm 0.2$ & & $0.4 \pm 0.4$ & $0.6 \pm 0.4$ & & & \\
\hline$x=0.16$ & $165 \pm 5$ & $710 \pm 42$ & $7.7 \pm 0.2$ & $7.8 \pm 0.2$ & $0.1 \pm 0.1$ & $1.2 \pm 0.4$ & $1.1 \pm 0.5$ & $1.12 \pm 0.06$ & $403 \pm 40$ & 4.24 \\
\hline$x=0.50$ & $175 \pm 5$ & $1070 \pm 63$ & $12.4 \pm 0.2$ & $12.0 \pm 0.2$ & $-0.4 \pm 0.1$ & $1.0 \pm 0.5$ & $1.4 \pm 0.5$ & $2.27 \pm 0.15$ & $541 \pm 60$ & \\
\hline
\end{tabular}

curves) were determined and are listed in Table I. From these two values, the perpendicular uniaxial magnetic anisotropy field $H_{2}\left(H_{2}=4 \pi M_{s}-4 \pi M_{\text {eff }}\right)$ was deduced and is also listed in Table I. Even though the error associated with $\mathrm{H}_{2}$ is rather large due to it being the difference of two comparable numbers, it is noteworthy that all films have a positive $\mathrm{H}_{2}$ value ranging from 0.5 to $1.2 \mathrm{kOe}$. This is probably associated with the residual strain in the films as indicated by XRD and/or with a surface-induced anisotropy as previously reported $^{12}$ in $\mathrm{Cu} / \mathrm{Ni} / \mathrm{Cu}(001)$ films.

The values of the magnetic exchange stiffness constant $A$ were determined from the temperature dependence of the saturation magnetization at low temperatures. According to the Bloch $T^{3 / 2}$ law, the ratio of the saturation magnetization $M_{s}(T)$ at low temperatures to the saturation magnetization $M_{s}(0)$ at absolute zero is given by ${ }^{13}$

$$
\frac{M_{s}(T)}{M_{s}(0)}=1-0.0587(Q s)^{1 / 2}\left(\frac{k_{B} T}{2 A a}\right)^{3 / 2},
$$

where $k_{B}$ is Boltzmann's constant, $Q$ the number of lattice points per unit cell ( $Q=4$ for fcc lattices), and $a$ the lattice constant. The spin quantum number $s$ is given by ${ }^{8}$

$$
s=\frac{M_{s}(0) a^{3}}{Q g \mu_{B}} .
$$

From the slopes of $M_{s}(T) / M_{s}(0)$ vs $T^{3 / 2}$ as shown in Fig. 4,

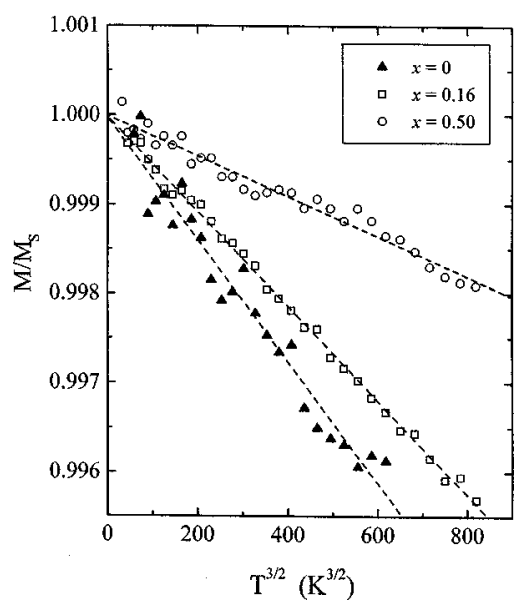

FIG. 4. Temperature dependence of the normalized saturation magnetization $M_{s}$ for the $\mathrm{Ni}_{1-x} \mathrm{Co}_{x}$ films. The dashed lines represent the $T^{3 / 2}$ fits to the data. the exchange stiffness constants were determined and found to increase from $(0.82 \pm 0.06) \times 10^{-6}$ to $(2.27 \pm 0.15)$ $\times 10^{-6} \mathrm{erg} / \mathrm{cm}$ as the Co concentration increases. These determinations as well as the resulting values for the spin-wave stiffness constant $D$ are listed in Table I.

The $D$ value obtained for our pure $\mathrm{Ni}$ film (449 $\pm 55) \mathrm{meV}^{2}$ is within the experimental uncertainty of the values deduced on bulk $\mathrm{Ni}$ from previous magnetization measurements ${ }^{5}$ and from the neutron diffraction results ${ }^{6}$ as seen in Fig. 1, but almost a factor of 3 times larger than the value determined from SWR measurements ${ }^{4}$ on comparable thickness Ni films. While the neutron diffraction determinations show an increase of $D$ with increasing concentration of Co from zero to $50 \%$, our results on the Ni-Co alloy films display a concentration dependence more resembling the prior magnetization and SWR studies ${ }^{5}$ with our $D$ values for the alloy films lying between the magnetization and neutron scattering results. These variations in the values of $D$ from the different measurements probably arise from the processing conditions and the structural quality of the films versus the bulk materials. Furthermore, as will be shown in the next section, the surface of the films plays an important role in the determination of $D$ from spin-wave resonance studies and may account for the significantly lower $D$ values deduced from the earlier SWR study ${ }^{4}$ than the present study on similar thickness Ni-Co films.

\section{B. FMR/SWR measurements}

Since all three films showed strong microwave resonance absorption modes, out-of-plane angular-dependent ferromagnetic resonance measurements could be performed. ${ }^{11}$ Multiple-resonance peaks were observed for the $x=0$ and $x=0.16$ films when the direction of the applied magnetic field approached that of the film normal. However, the sample with $x=0.50$ required fields beyond the limit of our electromagnet $(19 \mathrm{kOe})$ in order to track these modes completely. Figure 5 displays the FMR data (first derivative of the absorption spectrum) for the pure $\mathrm{Ni}$ film in the perpendicular geometry with multiple-resonance fields being clearly observable. These SWR data were subsequently analyzed following a model previously described by Soohoo, ${ }^{14}$ which assumes homogeneous magnetic properties throughout the films except at the surface, where the spins are (partially) pinned. 


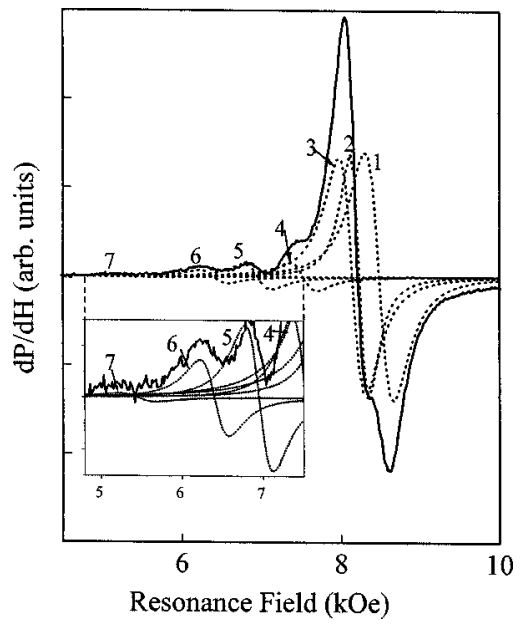

FIG. 5. Spin-wave resonance spectrum for the Ni film. The dotted lines represent the individual resonance peaks used to fit the overall spectrum.

In order to analyze the FMR/SWR data, the free energy density $E$ for a single-crystal film in the presence of a dc magnetic field $H$ is written as ${ }^{11}$

$$
\begin{aligned}
E= & -M_{s} H \sin \theta \cos \left(\phi-\phi_{H}\right)+\left(2 \pi M_{s}^{2}-K_{2}\right) \sin ^{2} \theta \sin ^{2} \phi \\
& -\frac{1}{2} K_{4}\left\{\sin ^{4} \theta\left(1-\sin ^{2} 2 \phi\right)+\cos ^{4} \theta\right\} .
\end{aligned}
$$

The three terms in Eq. (3) represent the Zeeman, effective demagnetization, and magnetocrystalline anisotropy contributions, respectively. $K_{2}$ and $K_{4}$ represent the second-order uniaxial perpendicular magnetic anisotropy and the fourthorder magnetocrystalline anisotropy constants, while $\theta$ and $\phi$ correspond to the polar and azimuthal orientations of the film's saturation magnetization with respect to a $x y z-$ coordinate system as described in Ref. 11. For the so-called out-of-plane FMR measurements, the film plane is kept parallel to the $x z$ plane while the dc magnetic field is applied parallel to the $x y$ plane, and $\phi_{H}$ is the angle the applied field makes with the $x$ axis.

The dispersion relation for microwave excited volume modes in a thin film is given by ${ }^{15}$

$$
\begin{aligned}
\left(\frac{\omega}{\gamma}\right)^{2}= & \left(\frac{1}{M_{s} \sin ^{2} \theta} \frac{\partial^{2} E}{\partial \phi^{2}}+\frac{2 A k^{2}}{M_{s}}\right)\left(\frac{1}{M_{s}} \frac{\partial^{2} E}{\partial \theta^{2}}+\frac{2 A k^{2}}{M_{s}}\right) \\
& -\left(\frac{1}{M_{s} \sin \theta} \frac{\partial^{2} E}{\partial \phi \partial \theta}\right)^{2},
\end{aligned}
$$

where $\omega=2 \pi f$ and $f$ is the microwave frequency. The parameters $\gamma$ and $k$ are the gyromagnetic ratio and the wave vector of the microwave field, respectively.

Under equilibrium conditions for the magnetization, outof-plane FMR measurements require $\partial E / \partial \theta=0$ and $\partial E / \partial \phi$ $=0$, which leads to $\theta=\pi / 2$ and

$$
H_{k} \sin \left(\phi-\phi_{H}\right)+\frac{1}{2}\left(4 \pi M_{\mathrm{eff}}\right) \sin 2 \phi+H_{4} \sin 4 \phi=0,
$$

where $H_{4}=2 K_{4} / M_{s}$ and the effective magnetic field $4 \pi M_{\text {eff }}=4 \pi M_{s}-2 K_{2} / M_{s}$.

By combining Eqs. (3), (4), and (5), the following expression is obtained:

$$
\begin{aligned}
\left(\frac{\omega}{\gamma}\right)^{2}= & \left\{H_{k} \cos \left(\phi-\phi_{H}\right)+4 \pi M_{\mathrm{eff}} \cos 2 \phi+H_{4} \cos 4 \phi\right. \\
& \left.+\frac{2 A k^{2}}{M_{s}}\right\} \times\left\{H_{k} \cos \left(\phi-\phi_{H}\right)-4 \pi M_{\mathrm{eff}} \sin ^{2} \phi\right. \\
& \left.+\frac{1}{2} H_{4}\left(2-\sin ^{2} 2 \phi\right)+\frac{2 A k^{2}}{M_{s}}\right\} .
\end{aligned}
$$

Note that the FMR mode, which is the resonance field $H_{0}$ corresponding to the uniform mode, can be obtained by setting $k=0$ in the above equations. The experimentally measured resonance fields can then be fitted to the theoretical expressions of Eqs. (5) and (6) in order to determine the values of $4 \pi M_{\text {eff }}, H_{2}$, and $H_{4}$. As seen in Table I these values from the FMR data are in good agreement with those deduced from the magnetization results on these films. Furthermore, the concentration variation in $H_{4}$ is in agreement with an earlier study on bulk $\mathrm{Ni}_{1-x} \mathrm{Co}_{x}$ alloys. ${ }^{16}$

For the perpendicular geometry $\left(\phi_{H}=\pi / 2\right)$, the equation for the resonance field corresponding to the $k$ th mode can be written as

$$
H_{k \perp}=-\frac{2 A k^{2}}{M_{s}}+H_{0 \perp},
$$

where $H_{0 \perp}$ corresponds to the uniform resonance mode [ $k$ $=0$ in Eq. (6)] and

$$
H_{0 \perp}=\frac{\omega}{\gamma}+4 \pi M_{\mathrm{eff}}-H_{4} .
$$

Obviously, either real or imaginary values for $k$ can satisfy Eqs. (6) and (7). Real values of $k\left(k^{2}>0\right)$ correspond to volume SWR modes with resonance fields that are lower than the uniform resonance field, whereas the imaginary values of $k\left(k^{2}<0\right)$ correspond to surface modes with resonance fields higher than the uniform resonance field. ${ }^{14}$ From the FMR spectrum shown in Fig. 5 as well as the spectra for the other Ni-Co films, the high-order resonance field values are clearly smaller than that of the uniform resonance mode and consequently these resonance modes correspond to volume SWR modes.

If the surface spins are completely pinned, $k=n \pi / d$, where $n$ is an integer known as the mode number and $d$ is the thickness of the film. Equation (7) now becomes

$$
H_{k \perp}=-\frac{2 A \pi^{2}}{M_{s} d^{2}} n^{2}+H_{0 \perp},
$$

which is known as Kittel's mode square law. ${ }^{13}$ However, for partial surface pinning of the spins, the so-called surface inhomogeneity (SI) model ${ }^{14,17}$ is more useful since it employs additional surface anisotropy energy and magnetization inhomogeneities close to the surface. The uniaxial surface anisotropy energy density with the easy axis normal to the film 


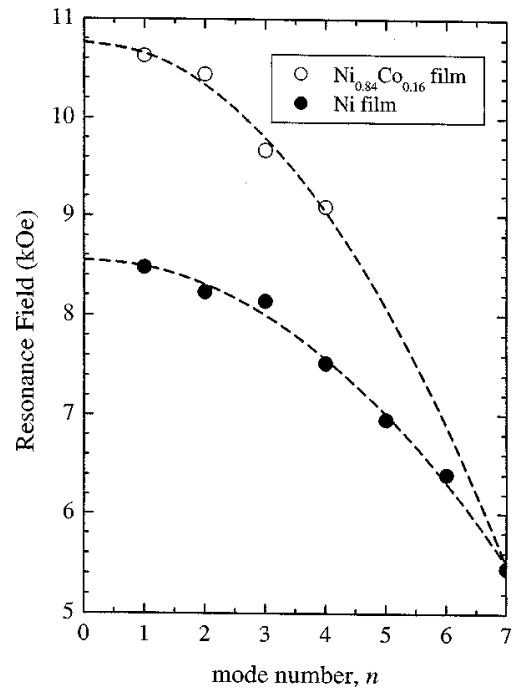

FIG. 6. Resonance fields as a function of the mode number $n$ as determined from the SWR spectra for the $\mathrm{Ni}_{0.84} \mathrm{Co}_{0.16}$ and pure $\mathrm{Ni}$ films. The symbols represent the experimental data, while the dashed lines are the least-squares-fitting curves to Eq. (7) for $K_{s}$ values of 4.24 and $0.93 \mathrm{erg} / \mathrm{cm}^{2}$ for the $\mathrm{Ni}_{0.84} \mathrm{Co}_{0.16}$ and pure $\mathrm{Ni}$ films, respectively.

plane is given by $E_{s}=-K_{s}^{\prime} \sin ^{2} \theta \sin ^{2} \phi$, where $K_{s}=K_{s}^{\prime} a$ defines the uniaxial surface anisotropy constant with $a$ being the lattice constant of the film. Assuming symmetrical spin pinning at both surfaces of the film, the locations of the spin-wave resonance modes for the applied field normal to the film plane $\left(\phi_{H}=\pi / 2\right)$ are given by ${ }^{14}$

$$
\tan k d=\frac{2 A k K_{s}}{(A k)^{2}-\left(K_{s}\right)^{2}} .
$$

Note that if the surface spins are completely pinned $\left(K_{s}\right.$ $\rightarrow \infty$ ), then Eq. (10) reduces to $k=n \pi / d$ where $n$ $=1,3,5, \ldots$. Since our films were grown with a $\mathrm{Cu}$ layer on both sides, symmetrical spin pinning at both surfaces is a reasonable assumption.

The resonance fields $H_{k \perp}$ were subsequently determined by fitting the first derivative of Lorentzian functions to the experimental FMR spectra. The resulting fits to the spectra (see Fig. 5 for the pure $\mathrm{Ni}$ spectrum and fit) indicate the presence of stronger resonance peaks for odd-numbered spin-wave modes and weaker resonance peaks for evennumbered modes. This means that the spins at the interfaces of the film are not strongly pinned. Figure 6 shows the resulting resonance field values determined from the fitting of the SWR spectra for the $x=0$ and 0.16 films. Although the resonance fields for the $\mathrm{Ni}_{0.84} \mathrm{Co}_{0.16}$ film are larger, the decrease in the resonance field with increasing mode number is a characteristic for both films. As there are more independent variables $\left(A, M_{s}, d, H_{0 \perp}\right)$ than the number of independent equations [Eqs. (7) and (10)] for fitting the resonance data, $K_{s}$ cannot be determined independently without using values for these variables deduced from other experimental measurements, e.g., from the magnetization measurements. Consequently, plots of $H_{k \perp}$ vs $(k d)^{2}$ (not shown) were generated for various $K_{s}$ values and then the slope from a least-squares fit was compared to $-2 A / M_{s} d^{2}$ [see Eq. (7)] for $A, M_{s}$, and $d$ values listed in Table I for each of these films. $K_{s}$ values of $0.93 \mathrm{erg} / \mathrm{cm}^{2}$ (for $x=0$ ) and $4.24 \mathrm{erg} / \mathrm{cm}^{2}$ (for $x=0.16$ ) were found to result in the best agreement. The dashed lines in Fig. 6 are the least-squares fit to Eq. (7) for these $K_{s}$ values as a function of mode number $n$. Although the experimental uncertainty in $H_{k}, A, M_{s}$, and $d$ results in acceptable fits for surface anisotropy energy values in the range of $\frac{1}{4} K_{s}$ to $4 K_{s}$, the $K_{s}$ value for the $\mathrm{Ni}-\mathrm{Cu}$ interface for our pure $\mathrm{Ni}$ film is in good agreement with an earlier value of $0.88 \mathrm{erg} / \mathrm{cm}^{2}$ obtained by Bochi et al. ${ }^{12}$ on $\mathrm{Cu} / \mathrm{Ni} / \mathrm{Cu}(001)$ film structures. The surface anisotropy in these epitaxially grown films probably is the result of the $\mathrm{Cu} / \mathrm{Ni}$ interfacial magnetocrystalline anisotropy as previously reported in these $\mathrm{Cu} / \mathrm{Ni} / \mathrm{Cu}(001)$ film structures. ${ }^{12}$ Moreover, the surface anisotropy field $H_{s}$ $\left(=4 K_{s} / M_{s} d\right)$ determined from these $K_{s}$ values is in reasonable agreement with our experimentally determined $\mathrm{H}_{2}$ values (see Table I). Although the positive $K_{s}$ value should favor the magnetization being normal to the film surface, the demagnetization energy dominates for the film thickness used in this study such that the magnetization lies in the plane of the film. It is only for Ni films with thickness less than $20 \mathrm{~nm}$ that the easy axis of magnetization is actually found to be normal to the plane of the film. ${ }^{14,18}$ The $K_{s}$ value of $4.24 \mathrm{erg} / \mathrm{cm}^{2}$ deduced for the $\mathrm{Ni}_{0.84} \mathrm{Co}_{0.16}$ film is substantially larger than that for any other system reported so far as $K_{s}$ values around $1 \mathrm{erg} / \mathrm{cm}^{2}$ have been typically reported, e.g., $0.88 \mathrm{erg} / \mathrm{cm}^{2}$ for $\mathrm{Ni} / \mathrm{Cu}(001),{ }^{12} 0.97 \mathrm{erg} / \mathrm{cm}^{2}$ for $\mathrm{Co} /$ $\mathrm{Pt}(111)$, and $0.92 \mathrm{erg} / \mathrm{cm}^{2}$ for $\mathrm{Co} / \mathrm{Pd}(111) .{ }^{19}$ It should be noted that the latter two systems show spontaneous perpendicular anisotropy only for Co film thickness $<1.6 \mathrm{~nm}$. More conclusive evidence for this large $K_{s}$ value in $\mathrm{Ni}_{0.84} \mathrm{Co}_{0.16} / \mathrm{Cu}(001)$ films would be to study the thickness dependence of magnetic anisotropy as a spontaneous perpendicular anisotropy should be readily apparent at smaller thicknesses.

In comparison to our inclusion of partial surface pinning in the analysis of the volume SWR modes, the earlier SWR study of Cullis and Heath ${ }^{4}$ considered the resonance modes to follow a strict Kittel's mode square law of Eq. (9) with an effective zero surface anisotropy. A least-squares fit of our resonance field data to the mode number squared ( $n$ $=1,2,3, \ldots)$ results in calculated values for the spin-wave stiffness constant $D$ of 392 and $379 \mathrm{meV}^{2}$ for the $\mathrm{Ni}$ and $\mathrm{Ni}_{0.84} \mathrm{Co}_{0.16}$ films, respectively, or about by about $10 \%$ lower than the values from the magnetization determinations. Although these recomputed $D$ values are still not as small as those of the earlier SWR study on uncharacterized surfaces of Ni-Co films, it points out that the determination of the spin-wave stiffness constants and exchange stiffness constants from SWR data is dependent upon the nature of the film's surfaces as standing spin waves in resonance experiments are more influenced by surfaces than the traveling spin waves in bulk magnetization measurements.

\section{CONCLUSIONS}

Various magnetic parameters have been determined from magnetization and ferromagnetic resonance measurements 
on epitaxial films of fcc $\mathrm{Ni}_{1-x} \mathrm{Co}_{x}(100)(x=0,0.16$, and 0.50) grown by MBE deposition on $\mathrm{Cu}(001) / \mathrm{Si}(001)$ substrates. By fitting the low-temperature saturation magnetization to the Bloch $T^{3 / 2}$ law, the exchange stiffness constant $A$ was determined to increase from $0.82 \times 10^{-6} \mathrm{erg} / \mathrm{cm}$ for the $\mathrm{Cu}-\mathrm{Ni}$ interface of the pure $\mathrm{Ni}$ film to $2.27 \times 10^{-6} \mathrm{erg} / \mathrm{cm}$ for the film with $50 \%$ Co. These results are in quantitative agreement with the numerical values obtained from prior neutron diffraction and magnetization measurements on bulk samples. In addition, spin-wave resonance volume modes were observed in the FMR spectra for the $x=0$ and 0.16 films with surface anisotropy constants being found to be approximately 1 and $4 \mathrm{erg} / \mathrm{cm}^{2}$, respectively. These large values of the surface anisotropy probably arise from the interfacial magnetocrystalline anisotropy between the $\mathrm{Cu}$ and $\mathrm{Ni}$ or Ni-Co layers.

\section{ACKNOWLEDGMENT}

This work was supported by the National Science Foundation Grant No. DGE-9870720.
${ }^{1}$ A. Hubert and R. Schäffer, Magnetic Domains (Springer-Verlag, Berlin, 1998).

${ }^{2}$ A. Michels, J. Weissmuller, A. Wiedenmann, J. S. Pedersen, and J. G. Barker, Philos. Mag. Lett. 80, 785 (2000).

${ }^{3}$ J. Weissmuller, R. D. McMichael, A. Michels, and R. D. Shull, J. Res. Natl. Inst. Stand. Technol. 104, 261 (1999).

${ }^{4}$ I. G. Cullis and M. Heath, Solid State Commun. 23, 891 (1977).

${ }^{5}$ I. Maeda, H. Yamauchi, and H. Watanabe, J. Phys. Soc. Jpn. 40, 1559 (1976).

${ }^{6}$ K. Mikke, J. Jankowska, A. Modrzejewski, and E. Frikkee, Physica B 86-88, 345 (1977).

${ }^{7}$ M. Hinoul and J. Witters, Solid State Commun. 10, 749 (1972).

${ }^{8}$ R. Kimura and H. Nosé, J. Phys. Soc. Jpn. 17, 604 (1962).

${ }^{9}$ P. K. George and E. D. Thompson, Phys. Rev. Lett. 24, 1431 (1970).

${ }^{10}$ S. Wakoh, J. Phys. Soc. Jpn. 30, 1068 (1971).

${ }^{11}$ R. Naik, C. Kota, J. S. Payson, and G. L. Dunifer, Phys. Rev. B
48, 1008 (1993); R. Naik, A. Poli, D. McKague, A. Lukaszew, and L. E. Wenger, ibid. 51, 3549 (1995).

${ }^{12}$ G. Bochi, C. A. Ballentine, H. E. Inglefield, C. V. Thompson, and R. C. O'Handley, Phys. Rev. B 53, R1729 (1996); G. Bochi, C. A. Ballentine, H. E. Inglefield, C. V. Thompson, R. C. O'Handley, H. J. Hug, B. Stiefel, A. Moser, and H.-J. Güntherodt, ibid. 52, 7311 (1995).

${ }^{13}$ C. Kittel, Introduction to Solid State Physics, 6th ed. (Wiley, New York, 1986).

${ }^{14}$ R. F. Soohoo, Phys. Rev. 131, 594 (1963).

${ }^{15}$ L. J. Maksymowicz and D. Sendorex, J. Magn. Magn. Mater. 37, 177 (1983).

${ }^{16}$ L. W. McKeehan, Phys. Rev. 51, 136 (1937).

${ }^{17}$ H. Puszkarski, Prog. Surf. Sci. 9, 191 (1979).

${ }^{18}$ S. Hameed, P. Talagala, R. Naik, L. E. Wenger, V. M. Naik, and R. Proksch, Phys. Rev. B 64, 184406 (2001).

${ }^{19}$ B. Heinrich and J. F. Cochran, Adv. Phys. 42, 523 (1993). 